\title{
Detection of respiratory syncytial virus and rhinovirus in healthy infants
}

\author{
Kohei Hasegawa ${ }^{1 *} \oplus$, Rachel W. Linnemann ${ }^{2 \dagger}$, Vasanthi Avadhanula ${ }^{3}$, Jonathan M. Mansbach ${ }^{5}$, \\ Pedro A. Piedra ${ }^{3,4}$, James E. Gern ${ }^{6}$ and Carlos A. Camargo Jr. ${ }^{1}$
}

\begin{abstract}
Background: Despite the research importance of rhinovirus detection in asymptomatic healthy infants, the literature remains sparse.

Objective: To investigate the prevalence of respiratory syncytial virus (RSV) and rhinovirus (and its species).

Methods: We conducted a cross-sectional study of 110 healthy, non-hospitalized infants without acute illness at an academic medical center from November 2013 through May 2014. We tested nasal swab specimens by using polymerase chain reaction and genetic sequencing.

Results: Overall, the median age was 3.8 months (IQR 2.0-5.1 months), $56 \%$ were male, and $90 \%$ were born $>37$ weeks. RSV was detected in nasal swabs from infants (1.8\%). By contrast, rhinovirus was detected in nasal swabs from 16 infants (14.5\%). Molecular typing assay revealed rhinovirus species: six rhinovirus-A (5.5 \%), one rhinovirus-B (0.9\%), eight rhinovirus-C (7.3\%), and one untypeable (0.9\%).

Conclusions: In this cross-sectional study of healthy, community-based infants, RSV was rare (<2\%) in nasal swabs, while rhinovirus was detected in $14.5 \%$ with a predominance of rhinovirus-A and $-C$. These finding are important for understanding the clinical significance of rhinovirus detection among infants hospitalized for bronchiolitis.
\end{abstract}

Keywords: Respiratory syncytial virus, Rhinovirus, Healthy infants, Asymptomatic, Prevalence

\section{Background}

Respiratory syncytial virus (RSV) and rhinovirus are the most frequently detected viral pathogens in infants with bronchiolitis, a leading cause of emergency department visit $[1,2]$ and hospitalization for United States infants [3]. Although it has long been the conventional wisdom that the infectious etiology of bronchiolitis does not affect outcomes, a growing number of studies have linked viral pathogens of bronchiolitis (e.g., rhinovirus) to long-term respiratory outcomes [4]. For example, studies reported that RSV bronchiolitis in infancy, especially when severe (e.g., requiring hospitalization) contribute to subsequent development of childhood asthma, while

\footnotetext{
*Correspondence: khasegawa1@partners.org

${ }^{\dagger} \mathrm{K}$. Hasegawa and R. W. Linnemann contributed equally to this work

1 Department of Emergency Medicine, Massachusetts General Hospital, Harvard Medical School, 125 Nashua Street, Suite 920, Boston, MA 02114, USA

Full list of author information is available at the end of the article
}

more recent research has focused on the potential role of rhinovirus bronchiolitis, particularly rhinovirus species $\mathrm{C}$, in early life in asthma pathogenesis [4].

A causal role of RSV and rhinovirus in lower respiratory infection is supported by isolation of viruses from the respiratory tract of children. However, advances in viral detection techniques (e.g., polymerase chain reaction [PCR] testing) have enabled increased identification of RSV and rhinovirus, not only in infants with acute respiratory infection (ARI) but also in asymptomatic individuals [5, 6]. A systematic review reported, in the respiratory samples of asymptomatic individuals (all ages), a mean viral prevalence of $2.6 \%$ for RSV and $15.1 \%$ for rhinovirus [5]. This finding questions the clinical significance of rhinovirus detection in young children with bronchiolitis. However, only a few studies, with small sample sizes, have specifically examined community-based infants (aged $<1$ year) without comorbidities and without a high risk for atopy $[7,8]$. Additionally, the 
prevalence of different rhinovirus species in this population remains largely unclear. To address these knowledge gaps, we investigated the prevalence of RSV and rhinovirus (and its species) by using PCR and molecular typing assay on nasal swab specimens from healthy, non-hospitalized infants without acute illness.

\section{Methods \\ Study design and participants}

We conducted a cross-sectional study of healthy infants enrolled from a primary care group practice at Massachusetts General Hospital (Boston, Massachusetts) from November 2013 through May 2014. Inclusion criteria were infants aged $<1$ year and gestational age $>34$ weeks. In the 3 days before a scheduled clinic visit, families were screened via phone to determine interest and eligibility. Exclusion criteria included comorbidities (heart-lung disease, immunodeficiency, immunosuppression, or chronic gastrointestinal disorder); previous lower respiratory infection that resulted in an urgent clinic visit, emergency department visit, or hospitalization; any diarrheal illness lasting $>24 \mathrm{~h}$ in the past week; any treatment with antibiotics in the past week; and current fever, ARI, or gastrointestinal illness. We defined current ARI and gastrointestinal illness based on the literature [9]. ARI was defined as two of the following symptoms for 1 day, or one of the following symptoms for two consecutive days: runny nose, stuffy or blocked nose, cough, fever, sore throat, or sneezing. Gastrointestinal illness was defined as: two or more looser than normal stools or any vomiting within $24 \mathrm{~h}$. The institutional review board of Massachusetts General Hospital approved the study. Informed consent was obtained from the parents or guardians.

\section{Data and sample collection}

Clinical data were collected from parents via structured interview and from medical record review. Data included infants' demographic characteristics as well as medical, environmental, and family history. Nasal swabs were collected from the anterior nares using a standardized protocol. Both nares were swabbed with a single nylon, pediatric FLOQSwab (Copan, Brescia, Italy). The swab was then added to $2 \mathrm{~mL}$ of viral transport medium and frozen at $-80{ }^{\circ} \mathrm{C}$. Samples were shipped on dry ice to the laboratory at Baylor College of Medicine, where they were stored at $-80{ }^{\circ} \mathrm{C}$. Samples were extracted and cDNA was generated using random hexamers for RSV and gene specific primers for rhinovirus. Singleplex and duplex real time PCR were used to detect rhinovirus and RSV respectively. Details of rhinovirus primers and probes have been described elsewhere [10], and sequences of RSV primers and probes are available upon request. Next, rhinovirus-positive specimens were sent to University of Wisconsin. Rhinovirus species were identified by using molecular typing assay that targets a variable fragment in $5^{\prime}$ untranslated region of the viral genome flanked by highly conserved motifs [11].

\section{Statistical analyses}

All analyses were performed using Stata SE 13.1 software (Stata Corp, College Station, TX). Patient demographic and clinical characteristics were compared by rhinovirus status, using Mann-Whitney, Chi Square, or Fisher exact tests, as appropriate.

\section{Results}

Table 1 summarizes demographic and clinical characteristics of the 110 enrolled infants. Overall, the median age was 3.8 months (IQR 2.0-5.1 months), $56 \%$ were male, $51 \%$ were non-Hispanic white, and $90 \%$ were born $>37$ weeks.

RSV was detected in nasal swabs from only two infants $(1.8 \%)$. By contrast, rhinovirus was detected in nasal swabs from 16 infants $(14.5 \%)$ with a median cycle threshold value of 29 (IQR 26-34). Molecular typing assay revealed rhinovirus species: six rhinovirus-A (5.5\%), one rhinovirus-B (0.9\%), eight rhinovirus- $\mathrm{C}(7.3 \%)$, and one untypeable $(0.9 \%)$. Infants with detected rhinovirus were older than those without (4.3 vs. 2.6 months; $\mathrm{P}=0.03$ ), and were more likely to attend daycare ( 43.8 vs. $9.6 \%$; $=0.002$ ) and be exposed to passive smoke in home ( 12.5 vs. $2.1 \%$; $=0.04)$. There were no other significant differences in demographic or clinical characteristics between the two groups (Table 1).

In a sensitivity analysis, we limited our analysis to infants who were completely symptom-free at the time of the screening phone call and enrollment. In this subset of 96 infants, RSV was detected in one infant (1.0\%) and rhinovirus in $11(11.5 \%)$. Molecular typing assay revealed six rhinovirus-A (6.3\%), no rhinovirus- $\mathrm{B}$, four rhinovirus- $\mathrm{C}(4.2 \%)$, and one untypeable (1.1\%).

\section{Discussion}

In this study of 110 healthy, community-based infants, RSV was rarely detected (1.8\%). By contrast, rhinovirus was detected in the nasal swab specimens from $14.5 \%$ of healthy infants, with predominance of rhinovirus-A and -C. When limiting our analysis to those who were completely asymptomatic at screening and enrollment, the rhinovirus prevalence decreased to $11.5 \%$.

Despite the research importance of rhinovirus (overall and species) detection in asymptomatic "healthy" infants, the literature remains sparse $[12,13]$. Our rhinovirus findings are in agreement with a prior U.S. study on asymptomatic infants ( $0-5$ months), which found overall rhinovirus prevalence of $11.4 \%$ with a rhinovirus-A and 
Table 1 Demographic and clinical characteristics of 110 healthy infants

\begin{tabular}{|c|c|c|c|c|}
\hline Characteristics & $\begin{array}{l}\text { All infants } \\
(n=110)\end{array}$ & $\begin{array}{l}\text { Rhinovirus positive } \\
(n=16)\end{array}$ & $\begin{array}{l}\text { Rhinovirus negative } \\
(\mathrm{n}=94)\end{array}$ & $P$ value \\
\hline \multicolumn{5}{|l|}{ Demographics characteristics } \\
\hline Age in months, median (IQR) & $3.8(2.0-5.1)$ & $4.3(3.6-5.8)$ & $2.6(1.9-4.5)$ & 0.03 \\
\hline Sex & & & & 0.60 \\
\hline Male & $62(56)$ & $10(63)$ & $52(55)$ & \\
\hline Female & $48(44)$ & $6(38)$ & $42(45)$ & \\
\hline Race/ethnicity & & & & 0.80 \\
\hline Non-hispanic white & $56(51)$ & $10(63)$ & $46(49)$ & \\
\hline Non-hispanic black & $11(10)$ & $1(6)$ & $10(11)$ & \\
\hline Hispanic & $20(18)$ & $3(19)$ & $17(18)$ & \\
\hline Other & $23(21)$ & $2(13)$ & $21(22)$ & \\
\hline Insurance & & & & 0.30 \\
\hline Private & $89(81)$ & $15(94)$ & $74(79)$ & \\
\hline Public & $21(19)$ & $1(6)$ & $20(21)$ & \\
\hline \multicolumn{5}{|l|}{ Home environment characteristics } \\
\hline Sibling in home & $47(43)$ & $7(44)$ & $40(43)$ & 0.93 \\
\hline Day care attendance & $16(15)$ & $7(44)$ & $9(10)$ & 0.002 \\
\hline Exposure to passive smoke in home & $4(4)$ & $2(13)$ & $2(2)$ & 0.04 \\
\hline \multicolumn{5}{|l|}{ Clinical characteristics } \\
\hline Gestational age & & & & 0.30 \\
\hline$\geq 40$ weeks & $46(42)$ & $4(25)$ & $42(45)$ & \\
\hline 38-39 weeks & $53(48)$ & $10(63)$ & $43(46)$ & \\
\hline 34-37 weeks & $11(10)$ & $2(13)$ & $9(10)$ & \\
\hline Delivery mode & & & & 0.92 \\
\hline Vaginal delivery & $70(64)$ & $10(63)$ & $60(64)$ & \\
\hline Cesarean section & $40(36)$ & $6(38)$ & $34(36)$ & \\
\hline $\begin{array}{l}\text { Primarily breast milk feeding from ages } 0 \text { to } \\
3 \text { months }\end{array}$ & $84(76)$ & $12(75)$ & $72(77)$ & 0.99 \\
\hline Eczema & $17(15)$ & $4(25)$ & $13(14)$ & 0.27 \\
\hline
\end{tabular}

Data were expressed as numbers (percentages) unless otherwise indicated

-C predominance (rhinovirus-A $7.0 \%$, rhinovirus-B $0 \%$, and rhinovirus-C $4.4 \%$ ) [12]. However, the earlier study differed by including children with comorbidities such as wheezing, chronic heart-lung disease, and immunodeficiency. In addition, other community-based studies were conducted among infants at high risk for atopy, including a Dutch study that used nasal brush specimens and found a rhinovirus prevalence of $17 \%$ among 6 month old asymptomatic infants, the majority of whom had a parent with allergic disease [5]. Likewise, in the Childhood Origins of Asthma study, the rhinovirus prevalence was $32 \%$ using nasal washes among infants $<1$ year, all of whom were at high risk for atopy [5]. Additional studies among healthy, community-based infants age $<1$ year using nasopharyngeal swabs or nasal washes reported rhinovirus prevalence of $12-24 \%$, but based on small samples ( $\mathrm{n}<35$ subjects) [7, 8]. A major strength of our study, compared to previous research, is assessing viral prevalence and rhinovirus species in a young population (median age 3.8 months, comparable to the median age of bronchiolitis hospitalization [4]) without comorbidities or high-risk of atopy.

Interestingly, we found that the rhinovirus genomic load among infants with rhinovirus detection was intermediate [14]. The mechanisms of rhinovirus positivity are unclear and likely multifactorial-e.g., active replication without respiratory symptoms, prolonged viral shedding, delayed clearance, and combinations of these factors in the population. The observed difference in patient characteristics (e.g. a higher rate of day care attendance and exposure to passive smoke in home among infants with rhinovirus detection) may paly a role in these potential mechanisms. The significance and mechanisms of rhinovirus detection in asymptomatic children merit further investigation.

Our finding of low RSV prevalence in asymptomatic infants suggests that RSV is likely the causative agent 
when detected in the setting of clinical symptoms of bronchiolitis. By contrast, given the observed prevalence of rhinovirus in infants without ARI, and even in infants without any respiratory symptoms, it remains unclear if rhinovirus detection always reflects current illness. Rhinovirus is frequently detected in the setting of multiple coexisting viruses [4]. Although a recent study of healthy infants showed that persistence of rhinovirus genome beyond 30 days was uncommon, $8.9 \%$ of distinct rhinovirus infections lasted longer than 14 days, and $4.5 \%$ lasted longer than 30 days [15]. Thus, while rhinovirus detected during bronchiolitis hospitalization can have an etiologic role, an alternate scenario is that the detected rhinovirus is persistent from a previous infection, and perhaps a marker of subject susceptibility or impaired immune response to rhinovirus infection. The generally higher rhinovirus prevalence found in studies of asymptomatic infants at high risk for atopy [5] may support this theory.

Our study has several potential limitations. First, the sample size, although larger than several prior studies, remains relatively small and all infants were recruited from one group practice in Boston, which may limit our generalizability to other settings. Second, this study was a single-year study; therefore, it is possible that an outbreak of a less virulent strain of rhinovirus occurred during this study period. Additionally, specimens were collected from late fall to early spring, yet rhinovirus infections occur year-round. While the observed prevalence could be different in months we did not enroll subjects, this chosen study period is helpful for bronchiolitis researchers. Third, ours study did not test other respiratory viruses, such as adenovirus, coronavirus, and human metapneumovirus. However, RSV and rhinoviruses are the most frequently detected respiratory viruses in young children (e.g., $85 \%$ in children hospitalized for bronchiolitis [4]). Lastly, this cross-sectional study did not follow up the infants after enrollment to determine if some went on to develop ARI in the days after sample collection. Nevertheless, our results add to a surprisingly sparse literature and will aid interpretation of studies on viral bronchiolitis.

\section{Conclusions}

In summary, in this cross-sectional study of healthy, community-based infants age $<1$ year without ARI or comorbidities, RSV was rare $(<2 \%)$ in nasal swabs, while rhinovirus was detected in $11-15 \%$ with a predominance of rhinovirus-A and -C. These finding are important for understanding the clinical significance of rhinovirus detection among infants hospitalized for bronchiolitis. To help tease out the role of rhinovirus detection in severe bronchiolitis and asthma pathogenesis, we plan to pursue future studies comparing rhinovirus prevalence, including genotyping, between infants hospitalized for severe bronchiolitis and healthy, community-based controls.

\section{Abbreviations}

ARI: acute respiratory infection; PCR: polymerase chain reaction; RSV: respiratory syncytial virus.

\section{Authors' contributions}

$\mathrm{KH}, \mathrm{RWL}$, and CAC contributed to the conception and design of this study; $\mathrm{KH}$ and RWL performed the statistical analysis. KH and RWL drafted the manuscript; VA, JMM, PAP, JEG critically reviewed the manuscript; CAC supervised the whole study process. Drs. KH and RWL contributed equally to this manuscript. All authors read and approved the final manuscript.

\section{Author details}

${ }^{1}$ Department of Emergency Medicine, Massachusetts General Hospital, Harvard Medical School, 125 Nashua Street, Suite 920, Boston, MA 02114, USA. 2 Division of Pediatric Pulmonology, Department of Pediatrics, Massachusetts General Hospital, Harvard Medical School, Boston, MA, USA. ${ }^{3}$ Departments of Molecular Virology and Microbiology, Baylor College of Medicine, Houston, TX, USA. ${ }^{4}$ Departments of Molecular Virology and Microbiology and Pediatrics, Baylor College of Medicine, Houston, TX, USA. ${ }^{5}$ Department of Medicine, Boston Children's Hospital, Harvard Medical School, Boston, MA, USA. ${ }^{6}$ Departments of Pediatrics and Medicine, University of Wisconsin School of Medicine and Public Health, Madison, WI, USA.

\section{Acknowledgements}

The authors extend sincere appreciation to all of the study families, as well as the physicians and staff at the Massachusetts General Hospital Pediatric Group Practice. This study was supported by the grants U01 Al-67693, R01 Al-114552, and R21HL129909 from the National Institutes of Health (Bethesda, MD). The content of this manuscript is solely the responsibility of the authors and does not necessarily represent the official views of the National Institute of Allergy and Infectious Diseases or the National Institutes of Health.

\section{Competing interests}

Dr. Mansbach has provided bronchiolitis-related consultation for Regeneron. Dr. Piedra has received grants from Gilead and provided bronchiolitis-related consultation for Alios, Gilead, Regeneron, and Teva. The other authors have no financial relationships relevant to this article to disclose.

Received: 10 October 2015 Accepted: 13 November 2015

Published online: 25 November 2015

\section{References}

1. Hasegawa K, Tsugawa Y, Cohen A, Camargo CA Jr. Infectious diseaserelated emergency department visits among children in the US. Pediatr Infect Dis J. 2015;34:681-5.

2. Hasegawa K, Tsugawa Y, Brown DF, Mansbach JM, Camargo CA Jr. Temporal trends in emergency department visits for bronchiolitis in the United States, 2006-2010. Pediatr Infect Dis J. 2014;33:11-8.

3. Hasegawa K, Tsugawa Y, Brown DF, Mansbach JM, Camargo CA Jr. Trends in bronchiolitis hospitalizations in the United States, 2000-2009. Pediatrics. 2013;132:28-36.

4. Hasegawa K, Mansbach JM, Camargo CA Jr. Infectious pathogens and bronchiolitis outcomes. Exp Rev Anti Infect Ther. 2014;12:817-28.

5. Jartti T, Jartti L, Peltola V, Waris M, Ruuskanen O. Identification of respiratory viruses in asymptomatic subjects: asymptomatic respiratory viral infections. Pediatr Infect Dis J. 2008;27:1103-7.

6. Chonmaitree T, Alvarez-Fernandez $P$, Jennings $K$, et al. Symptomatic and asymptomatic respiratory viral infections in the first year of life: association with acute otitis media development. Clin Infect Dis. 2015;60:1-9.

7. Fry AM, Lu X, Olsen SJ, et al. Human rhinovirus infections in rural Thailand: epidemiological evidence for rhinovirus as both pathogen and bystander. PLoS One. 2011;6:e17780. 
8. Jansen RR, Wieringa J, Koekkoek SM, et al. Frequent detection of respiratory viruses without symptoms: toward defining clinically relevant cutoff values. J Clin Microbiol. 2011;49:2631-6.

9. Lee GM, Salomon JA, Friedman JF, et al. Illness transmission in the home: a possible role for alcohol-based hand gels. Pediatrics. 2005;115:852-60.

10. Lu X, Holloway B, Dare RK, et al. Real-time reverse transcription-PCR assay for comprehensive detection of human rhinoviruses. J Clin Microbiol. 2008:46:533-9.

11. Bochkov YA, Grindle K, Vang F, Evans MD, Gern JE. Improved molecular typing assay for rhinovirus species A, B, and C. J Clin Microbiol. 2014;52:2461-71.

12. Iwane MK, Prill MM, Lu X, et al. Human rhinovirus species associated with hospitalizations for acute respiratory illness in young US children. Infect Dis J. 2011;204:1702-10.
13. Muller L, Mack I, Tapparel C, et al. Human rhinovirus types and association with respiratory symptoms during the first year of life. Pediatr Infect Dis J. 2015;34(8):907-9. doi:10.1097/INF.0000000000000758.

14. Jartti T, Hasegawa K, Mansbach JM, Piedra PA, Camargo CA Jr. Rhinovirusinduced bronchiolitis: lack of association between virus genomic load and short-term outcomes. J Allergy Clin Immunol. 2015;136(509-12):e11.

15. Loeffelholz MJ, Trujillo R, Pyles RB, et al. Duration of rhinovirus shedding in the upper respiratory tract in the first year of life. Pediatrics. 2014;134:1144-50.

\section{Submit your next manuscript to BioMed Central and we will help you at every step:}

- We accept pre-submission inquiries

- Our selector tool helps you to find the most relevant journal

- We provide round the clock customer support

- Convenient online submission

- Thorough peer review

- Inclusion in PubMed and all major indexing services

- Maximum visibility for your research

Submit your manuscript at www.biomedcentral.com/submit
() Biomed Central 